\title{
Psychological factors in recurrent genital herpes
}

Review

John Green, Agnes Kocsis

Objectives: To review recent research into psychological aspects of genital herpes and assess possible implications for clinical practice.

Methods: Review of all papers in the field on Medline 1985-96.

Results: Much attention has been paid to possible links between stress and recurrent genital herpes. There is no convincing evidence that stress in itself causes recurrences. It may be that recurrences are preceded by a prodromal period of altered mood. Patients with recurrences show considerable stress as a result of the disease, although most individuals eventually adjust psychologically. The impact of social support on adjustment remains unclear. The little evidence available suggests that antiviral treatments can help adjustment and the effects may perhaps outlast the period of active treatment.

Conclusions: Existing research gives some clues to optimal patient management but there is a need for future research to focus much more clearly on clinical issues, particularly on means of alleviating psychological distress and on the impact of antivirals.

(Genitourin Med 1997;73:253-258)

Keywords: genital herpes; herpes simplex virus; psychology; stress

\section{Introduction}

For many patients with recurrent genital herpes the psychological impact of having the disease overshadows the actual physical morbidity associated with it. Recurrences can be painful, even debilitating, but genital herpes tends to have few serious long term physical consequences for a man or woman, with the exception of the possibility of infection of the neonate. Such infections are serious but, with good management of the pregnant woman, rather rare. ${ }^{1}$ Treatment of recurrent genital herpes with antivirals in clinical practice is often undertaken in order to improve the quality of life of the patient and considerations of the impact of the disease on the mental wellbeing of the patient loom large in decisions about whether to initiate therapy. It is therefore important to consider what information is available about psychological aspects of recurrent genital herpes and the implications this information has for clinical practice. Accordingly, we searched Medline for all relevant papers published between 1985 and 1996. References cited in those papers and reviews by other authors were used to identify research of interest published before 1985 .

Since different researchers have approached the issue in different ways it is helpful to break down the literature into studies aimed at eliciting different information, although inevitably areas overlap and researchers have sometimes had multiple objectives.

\section{Investigations of possible causative effects of stress}

Most patients with recurrent herpes attacks identify stress as being a key element in causing recurrences of genital herpes. ${ }^{2-4}$ However, the belief that stress leads to all sorts of ills is common in the population at large. Reviewing the literature up to 1985 Vander Plate and $\mathrm{Aral}^{5}$ thought that "Although emotional stress is identified by many physicians and patients as a significant precipitant of genital herpes recurrence, data on that relationship are inconclusive ..." (p 69). The situation has not changed a great deal in the past decade.

There is evidence that those with high levels of recurrence are more likely to be under stress than those with less frequent recurrences. ${ }^{6}$ However, the high levels of stress might as easily be the result of the recurrences as their cause. People with more frequent attacks may be put under greater stress by their herpes and show more emotional distress, and perhaps more life events, as the attacks interfere with their performance at work and in their private lives.

Most studies which appear to show a relation between stress and recurrences are retrospective. Such studies are susceptible to systematic biases in recall and perception. For instance there is evidence that the severity of reported symptoms during herpes attacks, particularly pain, may be related more strongly to psychological factors than to actual physical parameters of the attack. ${ }^{7}$ Causality is almost always impossible to assess in this sort of design. Longo and $\mathrm{Clum}^{8}$ found a strong statistical relation between frequency of stressful experiences and frequency of recurrences in 46 individuals. Emotional distress was only weakly related to frequency of recurrences but was more strongly related to self rated severity of attacks. Hoon et al ${ }^{9}$ looked at 153 medical students seropositive for herpes. They did not find a direct influence of stress on recurrences but they did find that stress predisposed individuals to more general illnesses which, in turn, made recurrences of herpes more likely.

On the other hand, other authors using retrospective designs have failed to find an association of stress with recurrences. Silver et al ${ }^{10}$ reported that negative life stress was unrelated \\ Counselling Trai
}


to the frequency of attacks, although it was related to the duration of attacks. Frequency of attacks was related to how much respondents said the attacks bothered them. Overall, the sample reported high levels of emotional distress and worries about infecting sexual partners were very common.

Longitudinal prospective studies offer more promise in teasing out cause and effect in stress and herpes. Kemeny et al ${ }^{11}$ measured life events, mood, health behaviours and, in a proportion of cases, numbers of CD4+ and CD8+ $\mathrm{T}$ lymphocytes. While individuals who reported more stressful life events tended to have lower levels of CD4+ and CD8+ cells there was no direct impact of life events or moods on recurrences. Patients who were depressed and who did not report high levels of other illnesses did show more recurrences. However, depression in one month did not predict recurrences in the next month as might have been expected if there was a direct short term causal link.

Dalkvist et $a l^{12}$ followed 38 patients with genital herpes over a 3 month period using daily self reports. Recurrences were preceded by a period of decreased emotional wellbeing including increased levels of self rated nervousness and alertness lasting 4 and perhaps even 10 days. Their data raise the possibility that there might be a mood/cognitive prodrome in recurrent herpes which might be misinterpreted as stress provoking the attack. Their data are consistent with the results of McLarnon and Kaloupek ${ }^{13}$ who reported that recurrences were preceded by at least 4 days of elevated anxiety. Dalkvist et al were vague about possible linkages, suggesting prostaglandins as a possibility. However, other agents might be equally or more likely to have such an effect. Exogneous interferons are known to have marked, sometimes dramatic, neuropsychiatric effects, ${ }^{14}$ and an effect of endogenous interferon production in response to viral replication on psychological functioning seems at least a possibility. Herpes recurrence is regarded as a mainly local phenomenon with few systemic effects but this may be wrong. Patients report feeling ill during the attacks as their second most common problem. ${ }^{15}$

To complicate matters further, it is possible that some of the discrepancies in findings between studies may be the result of differences in samples. Vander Plate et al ${ }^{16}$ reported that there was a relation between retrospectively reported specific life events and stress in individuals who had had herpes recurrences for less than 4 years; however, for individuals with longer disease duration the effect disappeared. Herpes related social support, but not general social support, also appeared to affect the relation between recurrences and stressful life events. Recurrences were related to life events where such support was low. Patients' global ratings of stress over the past 12 months were positively related to number of recurrences. Once again, interpretation of the study is made more difficult because it was a retrospective study.

Overall, there is no convincing evidence that stress increases the rate of recurrences, although the possibility cannot be excluded. Most of the studies which appear to show an effect are also consistent with the hypothesis that more frequent recurrences put people under stress. The possibility that there is a prodrome involving low level cognitive and mood changes associated with herpes recurrences is one which deserves further investigation.

\section{Personality and herpes}

A related question which has attracted some attention is the question of whether some aspect of personality might be related to the development of, or severity of, recurrences. One study ${ }^{17}$ sought to establish whether type A behaviour pattern, commonly thought to be a factor in heart disease, might influence recurrences in patients with herpes. In fact type $A$ subjects did not differ from type $B$ subjects in frequency of recurrences, although they reported their recurrences to be more severe and sought more treatment. The authors attribute these results to possible immunological differences rather than differences in reporting of symptom severity with different personality types. However, the latter possibility seems more likely.

Goldmeier et $a l^{1819}$ reported on two studies in which a range of measures, including the Eysenck Personality Inventory, was used on samples of patients with herpes and the extent to which these predicted time to first recurrence was measured. There was little evidence that personality itself influenced recurrences. The main interest of the study came, however, not from the personality measures but from the data on distress. In the first study they found that scores on the General Health Questionnaire (GHQ) - a commonly used measure of psychological distress-was a significant predictor of time to first recurrence. In the second study there was a trend in that direction but the result was not significant. When the two studies were combined in a meta-analysis the effect for the GHQ was significant overall. As the authors note, it is possible that the differences reflected greater willingness to reattend in those with higher levels of psychological distress. GHQ scores can be affected directly by physical ill health so that it is possible that the link was mediated by some aspect of severity of first attack.

\section{Impact of herpes on psychological wellbeing}

The impact of having recurrent herpes on the psychological wellbeing of patients is fairly well attested. Goldmeier et $a l^{20}$ reported that patients with recurrent herpes were more distressed than those experiencing a first attack, although the effect was most marked for men and the authors remark on the difficulties in interpreting the GHQ in physically ill patients. Swanson et $a l^{21}$ reported that young adults with herpes had lower self esteem, more psychopathology, and reported more difficulties with everyday life than controls. 
Carney et $a l^{22}$ reported that patients with first attacks of genital herpes were highly distressed on the GHQ, more so than other STD patients and slightly, though not significantly, more so than dermatology controls. Scores on the Hospitals Anxiety and Depression Scale (HADS), a scale less responsive to physical ill health, failed to show any statistically significant differences between groups although the proportion of anxiety "cases" in the herpes group was $52 \%$ compared with $36 \%$ of other STD patients and $34 \%$ of dermatology controls.

Choice of an appropriate control group in such studies is a problem. One might argue about the value of proving that herpes patients were more or less anxious than those with nonspecific urethritis or gonorrhoea or other medical problems. The root question is to what extent patients with herpes are more distressed than they would have been if they had not had the disease and a case can be made for using a general population control, although this has its own difficulties. Those who did not have recurrences among the herpes patients showed a sizeable statistically significant reduction in GHQ levels and in HADS anxiety between initial visit and the second visit, 3 months later. However, making allowances for the use of "casedness" in the analysis which limits the power of statistics available, those who experienced recurrences appeared to show a smaller drop in distress both on the GHQ and the HADS anxiety scale than those who did not experience recurrences. One third of the herpes group had dropped out at 3 months and more than two thirds at 1 year. Dropout in this area is unlikely to be random, as the authors acknowledge, and those in greater distress may be more likely to stay in contact.

The same group ${ }^{23}$ reported that aciclovir reduced the patient's concern about the illness, and the the proportions classified as "cases" on the GHQ and HADS anxiety scales, although the loss of $40 \%$ of patients to follow up over a year introduces an element of uncertainty into the picture. Interestingly the improvement in wellbeing was maintained, at least in the short term, after cessation of aciclovir. Given the design, it is impossible to exclude time as a factor in the improvement seen. However, patients had had HSV for at least 1 year and showed a particularly sharp improvement in the first 3 months of taking the drug, suggesting a specific effect of being on medication. There is some evidence that HADS anxiety scores may decline as the length of time since the last herpes attack increases $^{24}$ and the reduction in frequency of attacks may well have been important. Other factors-for instance, increased feelings of control over the disease or an effect of feeling that the disease was being actively treated might also have been elements. There is a clear need to systematically explore the impact of drug treatments in genital herpes on psychological wellbeing through well designed randomised controlled trials.

There have been a number of studies of the impact of genital herpes on patients who have had herpes over fairly long period of time drawn from GUM clinics and self help groups. Both these groups are self selected and unlikely to be representative of those with recurrent herpes generally.

Such studies have identified a wide range of problems including difficulties with relationships with sexual partners and family and friends, fears of transmitting the virus to others, fears of possible long term consequences for general health, erectile dysfunction, problems with intimacy and spontaneity, and feelings of undesirability. ${ }^{25-27}$

However, Brookes et al, ${ }^{3}$ seeking to overcome the difficulties of such uncontrolled cross sectional designs, obtained self ratings on a variety of variables relative to before infection in long term herpes sufferers. The study had the problems of other retrospective data in this area. Overall, respondents identified surprisingly little change relative to before infection in self esteem, relationships with partners, friends and family, overall mood, or frequency or enjoyment of sex. Women reported more negative changes in these areas. A small number of individuals reported very high levels of distress even after several years.

In partial contrast, one study reported high levels of anxiety and sexual inhibitions among patients with herpes and that these were greater than before their infection. ${ }^{28}$ However, they did not differ from gonorrhoea patients on any of the measures. A large study of individuals who subscribed to a newsletter on herpes showed the readers reporting feelings of depression and fear of rejection. Sexual activity and enjoyment were also affected. ${ }^{29}$ It is likely that those who join self help groups or subscribe to newsletters on herpes differ from other individuals with herpes. ${ }^{30} \mathrm{It}$ is possible to speculate that such individuals might be those who are experiencing the greatest difficulty in adjustment to herpes, for whatever reason, or who have the most severe symptoms or who seek to deal with the disease by seeking out information about it.

\section{Mediators of the psychological response to herpes}

A number of authors have sought to establish why some individuals appear to react much more adversely psychologically to herpes than others.

One possibility is the coping strategies which patients with herpes use. One study ${ }^{10}$ reported that patients who had frequent severe recurrences were likely to view their lives as less under their control, to engage in more wishful thinking, and to be less likely to try to control their own thinking about the problem. Manne et $a l^{30}$ reported that patients who engaged in self blame, who brooded on their problem, and who engaged in wishful thinking were worse adjusted to their disease. Another indicator of poor adjustment was seeking medical and other treatments for the disease, including attending clinics, suggesting that samples of patients with recurrent herpes recruited in clinics may over-represent this group. 
Non-drug interventions in herpes

Given the psychological problems which can be associated with herpes the possibility of intervening directly to reduce patient distress is one which has immediate appeal. Given that herpes is still a stigmatised disease, social support might be expected to be an important element. Unfortunately, the literature gives conflicting results on the benefits of social support. People with greater social support are variously reported to have more recurrences, ${ }^{9}$ to have neither more nor more severe recurrences, ${ }^{8}$ to be no different in number of recurrences but to show lower psychopathology, ${ }^{10}$ and to have more recurrences where the number of life events is high. ${ }^{16}$ Unfortunately more effort seems to have gone into measuring the impact of social support on disease manifestations than on distress.

Membership of a self help group might be expected to improve functioning. However, in one study people enrolled in self help groups appeared to adjust worse to herpes and show more continuing distress than other patients..$^{30}$ In part, at least, this is probably the result of self selection by group members. Those who adjust least are perhaps most likely to stay in self help groups. However, the authors raise the possibility that self help groups may sometimes be unhelpful by encouraging the patient to focus on the disease and encouraging a search for an unavailable "cure" which may inhibit adjustment. If this is, in fact, so then presumably the approach taken by a particular group will determine whether its impact is positive or negative.

A few studies have looked at more systematic interventions. A small scale study compared a cognitive therapy group against a discussion group and found no difference in distress between the two groups after five sessions. However, the cognitive group showed fewer recurrences at follow up. ${ }^{13}$ Relaxation training has also been reported to reduce the frequency of recurrences. ${ }^{31}$

Clearly there is a need for more work to be done on establishing the best way to help people with recurrent herpes to adjust to the disease and to maximise their quality of life.

\section{Discussion}

The literature on herpes is rather disappointing; much effort has gone into the possible link with stress and less into more clinically relevant areas. For instance, few studies have anything to say about the key area of the impact of antiviral treatments on the psychological wellbeing of patients. It is vital that trials of antiviral drugs should attempt to address this issue with proper systematic measures. We know that many, if not most, individuals with recurrent herpes do adjust and that the long term impact on their lives is less than might be expected. We know little or nothing about the successful strategies which patients use in order to manage their disease. For example, we do not know how they deal with the negotiations around new partners, what in practice they do to minimise the risk of transmission and what are the most successful practical and psychological strategies for adapting to the disease. Nor does the literature clearly establish the benefits or otherwise of social support or answer the question as to whether we should be encouraging patients to join self help groups, or even to avoid them.

However, examination of the literature combined with what is known of psychological adjustment to illness generally does allow some tentative suggestions to be made about strategies which are likely to help rather than hinder the patient's adjustment. What follows is speculative but because the clinician is obliged to treat, even in the absence of conclusive research data in key areas, some degree of speculation is probably justified, if not inevitable.

Firstly, many patients feel that their level of stress leads to attacks, worry about this and their concerns then put them under even more stress. This can easily lead to them feeling that they are, in some way, failing and out of control. A similar phenomenon is seen in HIV. ${ }^{32}$ If there is an effect of stress then it is unlikely to be a major factor in the natural history of the disease. As, on current knowledge, it is more $\bigcirc$ likely that stress results from recurrences $\vec{P}$ rather than vice versa, we are in a position to reassure our patients about this.

Patients on diagnosis need clear, low key information covering the nature and natural $₫$ history of the disease and ways of protecting partners. Feeling in control is known to be important in adjustment to illness and having adequate correct information, particularly about what actions need to be taken, is known to be an important factor in gaining control. ${ }^{334}$ While not diminishing the patient's experience and their distress, it is important to stress that this is, when all is said and done, a cold sore and one which hundreds of thousands of people worldwide have contracted and coped with. It is important not to assume that patients start with no ideas about herpes. Patients are likely to have their own information about herpes, gleaned from out of date textbooks, self help books, rumour, and alarmist media presentations. In the past it was widely thought that herpes caused cervical carcinoma and many books still state this as a fact. The connection has moved in the literature over 20 years from direct causation to cofactor and, in at least one recent major study, ${ }^{35}$ has disappeared altogether.

We can reassure patients that most women with herpes can have a perfectly normal birth $^{36-38}$ and the chances of infection of the neonate, with good clinical management, should be remote. A man who has herpes and has a possible uninfected partner needs to be consistent either in avoiding intercourse or in using a condom during his partner's pregnancy since primary infection of the woman in the third trimester may raise the risk of infection of the neonate. This needs to be explained, particularly as a couple using condoms for contraception are probably least likely to do so when the woman is actually pregnant. 
Asymptomatic shedding-and inapparent recurrences-is a particularly difficult area. Infections do occur during asymptomatic shedding. ${ }^{39} 40$ Indeed, avoidance of sex during prodrome and attacks may increase the relative risk of this phenomenon. ${ }^{15}$ As viral detection assays improve in sensitivity it seems likely that more patients will be found to shed virus asymptomatically for more of the time. However, detecting virus at low titre on sensitive assays is not necessarily the same as the patient being infectious in the real world. Partner characteristics, including issues such as past infection with HSV-I, further complicate the picture. ${ }^{39}$ Patients need to be warned about the problem and the risks discussed. However, we do not wish to create an unwarranted feeling of continuous contamination in people with herpes. Most men and women with herpes are eventually going to want to have unprotected intercourse, if only to produce children. Striking a balance between scare and risk reduction is difficult.

Above all patients need help to try to solve some of the practical problems which herpes presents them with. For instance they may need to work out how to approach new partners-and existing partners, when to tell them and what to tell them. Informing family and friends may be an issue. Most clinicians will be familiar with the stories of many cases while the herpes patients will, as far as they are aware, know no one else with the disease. Simple steps, such as seeking to get to know potential partners well over a period of time and forming a strong relationship with them before contemplating sex can improve a patient's success enormously.

In longer term relationships with discordant couples who have engaged in unprotected intercourse it is entirely possible that a partner has already been asymptomatically infected. Until recently the lack of a reliable type specific antibody test for routine use has spared us some of the issues around such situations. A great deal of care and thought needs to go into the use of such tests given uncertainties about the significance of different viral genotypes and the significant proportion of genital herpes attributable to HSV-I infections. ${ }^{1}$ Testing of couples in order to assess possible transmission risks is clearly not to be entered into lightly although it may convey some benefits in some situations.

It appears, on clinical experience and on the basis of the rather thin research evidence, that antiviral drugs can play a part in reducing distress and that this effect may extend beyond active treatment, possibly because they provide patients with a "holiday" from attacks in which to adjust and a feeling of control over the disease. This is obviously an important consideration with a class of drugs which, while they have a good long term safety profile and few side effects, are expensive and do not affect the natural history of the disease.

Unfortunately it is unclear to what extent antiviral drugs affect transmission risks. ${ }^{41}$ There is a need for a study establishing the impact of antivirals on transmission of HSV, including asymptomatic transmission. Given an overall rate of transmission of the virus of perhaps $10 \%$ per annum within discordant couples $^{39}{ }^{40}$ and the need to instruct patients to reduce their behavioural risks as far as possible, such a study would need to be large and hence it would be expensive. However, it would be of enormous value to be able to help patients to reduce their risks of transmission, although a partial reduction in risk might be a mixed blessing by encouraging some patients to discontinue other risk reduction methods.

Herpes can cause enormous distress to patients. However, over time most patients appear to adjust. Patients who keeps coming back to a GUM clinic in distress with their disease, or who come back more than a year after diagnosis may well be experiencing particularly severe attacks or to be experiencing difficulties in adjusting. They need to be assessed for anxiety and depression and they need careful counselling to find out what problems they are having and whether further antiviral or counselling approaches need to be implemented. Overcounselling patients, particularly in the early stages of the disease, runs the danger of giving the message that herpes is a dreadful disease which they are not going to cope with. On the other hand it is important not to miss the patient in relievable distress. As usual in clinical matters, there is a fine and difficult balance to be struck.

1 Robertson DHH, McMillan A, Young H. Clinical practice in sexually transmissible diseases. Edinburgh: Churchil Livingstone, 1989

2 Swanson JM, Dibble SL. Genital herpes: clinical features, sources of information, recurrences and treatment in young adults. Dermatol Nurs 1993;5:365-73.

3 Brookes JL, Haywood S, Green J. Adjustment to the psychological and social sequelae of recurrent gential herpes simplex infection. Genitourin Med 1993;69:384-7.

4 Hamilton R. The herpes book. Boston: Houghton Mifflin, 1980.

5 Vander Plate C, Aral SO. Psychosocial aspects of genital herpes virus infection. Health Psychol 1987;6:57-72.

6 Stout CW, Bloom LJ. Genital herpes and personality. $\mathcal{F}$ Human Stress 1986;12:119-24.

7 Levenson JL, Hamer RM, Myers T, Hart RP, Kaplowitz LG. Psychological factors predict symptoms of severe recurrent genital herpes infection. $\mathcal{F}$ Psychosom Res 1987 31:153-9.

8 Longo DJ, Clum GA. Psychosocial factors affecting genital herpes recurrences; linear vs mediating models. $\mathcal{f}$ Psychosom Res 1989;33:161-6.

9 Hoon EF, Hoon PF, Rand KH, Johnson J, Hall NR, Edwards NB. A psycho behavioural model of genital herpes recurrence. F Psychosom Res 1991;35:25-36.

10 Silver PS, Auerbach SM, Vishniavsky N, Kaplowitz LG. Psychological factors in recurrent genital herpes infection: stress, coping style, social support, emotional dysfunction and symptom recurrence. $\mathcal{F}$ Psychosom Res 1986 30:163-71.

11 Kemeny ME, Cohen F, Zegans LS, Conaant MA Psychological and immunological predictors of genital herpes recurrences. Psychosom Med 1989;51:195-208.

12 Dalkvist J, Wahlin TB, Bartsch E, Forsbeck M. Herpes simplex and mood: a prospective study. Psychosom Med 1995;57:127-37.

13 McLarnon LD, Kaloupek DG. Psychological investigation of genital herpes recurrence: prospective assessment and of genital herpes recurrence: prospective assessment and
cognitive behavioural intervention for a chronic physical cognitive behavioural intervention for a
disorder. Health Psychol 1988;7:231-49.

14 Janssen HL, Brouwer JT, van der Mast RC, Schalm SW. Suicide associated with alpha interferon therapy for chronic viral hepatitis. F Hepatol 1994;21:241-3.

15 Brookes JL, Haywood S, Green J. Prodromal symptoms in genital herpes simplex infection. Genitourinary Med 1992; 68:347-8.

16 Vander Plate C, Aral SO, Magder L. The relationship among genital herpes simplex virus, stress and social support. Health Psychol 1988;7:159-68.

17 Lacroix JM, Offutt C. Type A and genital herpes. $f$ Psychosom Res 1988;32:207-12.

18 Goldmeier D, Johnson A. Does psychiatric illness affect the recurrence rate of genital herpes? Br $\mathcal{F}$ Vener Dis 1982; 58:40-3. 
19 Goldmeier D, Johnson A, Jeffries D, Walker GD, Underhill G, Robinson G, Ribbans H. Psychological aspects of recurrences of genital herpes. $\mathcal{F}$ Psychosom Res 1986;30: 601-8.

20 Goldmeier D, Johnson A, Byrne M, Barton S. Psychosocial implications of recurrent genital herpes simplex virus infection. Genitourin Med 1988;64:327-30.

21 Swanson JM, Dibble SL, Chenitz WC. Clinical features and psychosocial factors in young adults with genital herpes. Image ₹ Nurs Sch 1995;27:16-22.

22 Carney O, Ross E, Bunker C, Ikkos G, Mindel A. A prospective study of the psychological impact on patients with a first episode of genital herpes. Genitourin Med 1994;70:40-5.

23 Carney O, Ross E, Ikkos G, Mindel A. The effect of suppressive oral acyclovir on the psychological morbidity associated with recurrent genital herpes. Genitourin Med 1993;69:457-9.

24 Cassidy LC, Holder MA, Barton SE, Meadows J, Catalan J. Are doctors in genitourinary medicine clinics perceiving the psychological impact of recurrent genital herpes? Genitourin Med 1994;70:357-9.

25 Keller ML, Jadack RA, Mims LF. Perceived stressors and coping responses in persons with recurrent genital herpes. Res Nurs Health 1991;14:421-30.

26 Luby ED, Klinge V. Genital herpes. A pervasive psychosocial disorder. Arch Dermatol 1985;121:494-7.

27 Drob S, Loemer M, Lifschutz H. Genital herpes: the psychological consequences. $\mathrm{Br} f \mathrm{Med}$ Psychol 1985;58: 307-15.

28 Stronks DL, Rijpma SE, Passchier J. Verhage F, van der Meinjden W, Stoltz E. Psychological consequences of genital herpes, and exploratory study with gonorrhoea control group. Psychol Rep 1993;73:395-400.

29 Catotti DN, Clarke P, Catoe KE. Herpes revisited. Still a cause for concern. Sex Transm Dis 1993;20:77-80.

30 Manne S, Sandler I, Zautra A. Coping and adjustment to genital herpes: the effects of time and social support. $\mathcal{F}$

31 Koehn KA, Burnette MM, Stark C. Applied relaxation training in the treatment of genital herpes. $\mathcal{F}$ Behav Ther Exp Psychiatry 1993;24:331-41.

32 Green J, Hedge B. Psychological factors in HIV disease progression. In: Cooper C, ed. Cancer and stress. Oxford: Oxford Universitry Press, 1991.

33 Taylor SE. Adjustment to threatening events: a theory of cognitive adaptation. Am Psychol 1983;38:1161-73.

34 Thompson S, Nanni C, Levine A. Primary versus secondary and central versus consequence related control in HIV positive men. F Personal Soc Psychol 1994;67:540-7.

35 Lehtinen M, Dillner J, Knekt P, Luostarinen T, Aromaa A, Kirnbauer $R$, et al. Serologically diagnosed infection with human papillomavirus type 16 and risk for subsequent development of cervical carcinoma: nested case control study. $B M F$ 1996;312:537-9.

36 van Everdingen J, Peeters MF, ten Have P. Neonatal policy in the Netherlands. Five years after a concensus conference. F Perinatal Med 1993;21:371-5.

37 Forsoren $M$. Genital herpes simplex virus infection and incidence of neonatal disease in Sweden. Scand 7 Infect Dis Suppl 1990;69:37-41.

38 Roberts SW, Cox SM, Dax J, Wendel GD, Leveno KJ. Genital herpes during pregnancy, no lesions no caeGenital herpes during preg:85:261-4 lesions no cae-

39 Metz GJ, Benedetti J, Ashley RA, Selke SA, Corey L. Risk fact factors for the sexual transmission of genital herpes. Ann Intern Med 1992;116:197-202.

40 Bryson Y, Dillon M, Brenstein DI, Radolph J, Zakowski P, Garratty E. Risk of acquisition of genital herpes simplex virus type 2 in sex partners of persons with genital herpes: a prospective couple study. F Infect Dis 1993;116: 197-202.

41 Patel R, Cowan FM, Barton S. Advising patients with genital herpes. $B M \mathcal{F}$ 1997;314:85-6. 